\title{
MULTIPLICITIES AND GROTHENDIECK GROUPS $\left({ }^{1}\right)$
}

\author{
BY \\ MARSHALL FRASER
}

The concept of the multiplicity of a system of parameters of a local ring has played a central role in algebraic geometry. Recently the subject has received a homological treatment by Serre [7] and Auslander and Buchsbaum [1]. Also the notion of multiplicity has been generalized to a general multiplicity theory of Wright [8]. The general multiplicity can be thought of as follows: If $R$ is a commutative Noetherian ring, if $x_{1}, \ldots, x_{s}$ is a set of elements of $R$ satisfying the condition that $R /\left(x_{1}, \ldots, x_{s}\right)$ has finite length, and if $E$ is a finitely generated $R$-module, then a symbol $e_{R}\left(x_{1}, \ldots, x_{s} \mid E\right)$ is defined taking its values in the integers. The general multiplicity symbol is then a map from the category of finitely generated $R$-modules to the integers.

A fundamental property of the map $e_{R}\left(x_{1}, \ldots, x_{s} \mid E\right)$ is additivity on exact sequences; it is an Euler-Poincaré map. This suggests that Grothendieck groups may be an appropriate setting in which to define and study the multiplicity map. We do this in this paper. If $R$ is a commutative Noetherian ring and $x_{1}, \ldots, x_{s}$ is any set of elements of $R$ we define a multiplicity symbol $\chi_{R}\left(x_{1}, \ldots, x_{s}\right)$ which turns out to be a homomorphism from the Grothendieck group of the category of finitely generated $R$-modules to the Grothendieck group of the category of finitely generated modules over $R /\left(x_{1}, \ldots, x_{s}\right)$. This amounts to a generalization of the general multiplicity theory in that we need not assume $R /\left(x_{1}, \ldots, x_{s}\right)$ has finite length. However it poses the problem of finding appropriate statements about Grothendieck groups in which to express properties of multiplicities. This can usually be done and the proofs of these properties, since they depend upon general properties of Grothendieck groups, become simpler and more transparent.

This paper is divided into three sections. In $\S 1$ we define the multiplicity map and prove many of its fundamental properties. In $\$ 2$ we define the Hilbert function taking values in a Grothendieck group and explore the relation between it and the multiplicity map. In $\S 3$ we present some miscellaneous results.

Throughout this paper $R$ will denote a commutative Noetherian ring with unit. By module we will always mean a finitely generated unitary module. If $\mathscr{C}$ is an abelian category we denote the Grothendieck group of $\mathscr{C}$ by $K^{0}(\mathscr{C})$. For our purposes $\mathscr{C}$ is usually the category of all finitely generated $R$-modules. In this special

Received by the editors August 3, 1967 and, in revised form, December 9, 1967.

(1) This paper contains the results of the author's Ph.D. thesis, University of Illinois, 1967. I wish to thank Professor John Eagon for his suggestions and criticisms. Part of the work of this paper was supported by NSF Grant \# GP 5478. 
case we denote the Grothendieck group by $K(R)$. If $E$ is a module in $\mathscr{C}$ let $[E]$ denote the class of $E$ in $K^{0}(\mathscr{C})$. It is well known that the set $\{[R / P] \mid P$ prime in $R\}$ is a set of generators of $K(R)$. We let $Z(R)$ be the subgroup of $K(R)$ generated by the set $\{[R / P] \mid$ height $P>0\}$ and define $D(R)$ to be the free abelian group with basis $\{\langle P\rangle \mid$ height $P=0\}$. Then $K(R)=D(R) \oplus Z(R)$.

1. The general multiplicity map. In this section we define the general multiplicity map using the Koszul complex as in [1] and generalize appropriately its well-known properties which are found in [8].

Suppose $x_{1}, \ldots, x_{s}$ are elements of $R$ and let $I=\left(x_{1}, \ldots, x_{s}\right)$. The general multiplicity map will be denoted by $\chi_{R}\left(x_{1}, \ldots, x_{s}\right)$ and will be a homomorphism of $K(R)$ into $K(R / I)$. If $E$ is an $R$-module then $E_{x: 1, \ldots, s}$ denotes the Koszul complex of $E$ generated by $x_{1}, \ldots, x_{s}$ see $[1, \S 1]$ and $H_{i}\left(E_{x: 1, \ldots, s}\right)$ denotes the $i$ th homology module of the Koszul complex. Then $H_{i}\left(E_{x: 1, \ldots, s}\right)=0$ for $i<0$ and $i>s$ and $I \cdot H_{i}\left(E_{x: 1, \ldots, s}\right)=0$ for $i=0,1, \ldots, s\left[1\right.$, Proposition 1.5, p. 628]. So $H_{i}\left(E_{x: 1, \ldots, s}\right)$ is a module over $R / I$. Define

$$
\chi_{R}\left(x_{1}, \ldots, x_{s}\right): K(R) \rightarrow K(R / I)
$$

by $\chi_{R}\left(x_{1}, \ldots, x_{s}\right)[E]=\sum_{i=0}^{s}(-1)^{i}\left[H_{i}\left(E_{x: 1, \ldots, s}\right)\right]$. This map is well defined by the exact homology sequence established in [1, Proposition 1.5, p. 628]. If $s=0$ define $\chi_{R}(\cdot): K(R) \rightarrow K(R)$ to be the identity map. The case $s=0$ is a useful convention in what follows. We have the following propositions.

1.1. Proposition. Let $x_{1}, \ldots, x_{s}$ be elements of $R$. If $\pi$ is a permutation of $1, \ldots, s$ then

$$
\chi_{R}\left(x_{\pi 1}, \ldots, x_{\pi s}\right)=\chi_{R}\left(x_{1}, \ldots, x_{s}\right) .
$$

1.2. Proposition. If $x_{1}^{n} E=0$ for some $n$ then $\chi_{R}\left(x_{1}, \ldots, x_{s}\right)[E]=0[1$, Proposition 3.2, p. 635].

1.3. Proposition. If $x_{1}$ is not a zero-divisor on $E$ then $\chi_{R}\left(x_{1}, \ldots, x_{s}\right)[E]=$ $\chi_{R / x_{1} R}\left(\bar{x}_{2}, \ldots, \bar{x}_{s}\right)\left[E / x_{1} E\right]$, where $\bar{x}_{2}, \ldots, \bar{x}_{s}$ are the images of $x_{2}, \ldots, x_{s}$ in $R / x_{1} R$ and $\chi_{R / x_{1} R}\left(\bar{x}_{2}, \ldots, \bar{x}_{s}\right): K\left(R / x_{1} R\right) \rightarrow K(R / I)$ [1, Proposition 3.2, p. 635].

1.4. Proposition. $\chi_{R}\left(x_{1}, \ldots, x_{s}\right)[E]=\chi_{R / x_{1} R}\left(\bar{x}_{2}, \ldots, \bar{x}_{s}\right)\left[x_{1}^{n} E / x_{1}^{n+1} E\right]$ for all $n$ sufficiently large [1, Proof of Theorem 3.3, p. 636].

1.5. Corollary. If $x$ is an element of $R$ then in $K(R / x R)$ we have $\left[x^{n} E / x^{n+1} E\right]$ $=[E / x E]-\left[(0: x)_{E}\right]$ for all $n$ sufficiently large.

Proof. $\left[x^{n} E / x^{n+1} E\right]=\chi_{R}(x)[E]=\left[H_{0}\left(E_{x}\right)\right]-\left[H_{1}\left(E_{x}\right)\right]=[E / x E]-\left[(0: x)_{E}\right]$.

Putting Proposition 1.4 and Corollary 1.5 together we obtain the following theorem.

1.6. ThEOREM. Let $I=\left(x_{1}, \ldots, x_{s}\right)$. Then

$$
\chi_{R}\left(x_{1}, \ldots, x_{s}\right)[E]=\chi_{R / x_{1} R}\left(\bar{x}_{2}, \ldots, \bar{x}_{s}\right)\left[E / x_{1} E\right]-\chi_{R / x_{1} R}\left(\bar{x}_{2}, \ldots, \bar{x}_{s}\right)\left[\left(0: x_{1}\right)_{E}\right],
$$

where $\bar{x}_{2}, \ldots, \bar{x}_{s}$ are the images of $x_{2}, \ldots, x_{s}$ in $R / x_{1} R$ and

$$
\chi_{R / x_{1} R}\left(\bar{x}_{2}, \ldots, \bar{x}_{s}\right): K\left(R / x_{1} R\right) \rightarrow K(R / I) .
$$


We have defined a multiplicity map in terms of the Koszul complex, but we see that Theorem 1.6 and the following Corollary 1.7 embody the inductive definition of multiplicity as found in [8].

\subsection{Corollary. We have}

Further

$$
\chi_{R}\left(x_{1}, \ldots, x_{s}\right)=\chi_{R / x_{1} R}\left(\bar{x}_{2}, \ldots, \bar{x}_{s}\right) \cdot \chi_{R}\left(x_{1}\right) .
$$

$$
\chi_{R}\left(x_{1}, \ldots, x_{s}\right)=\chi_{R /\left(x_{1}, \ldots, x_{s-1}\right)}\left(\bar{x}_{s}\right) \cdot \chi_{R /\left(x_{1}, \ldots, x_{s}\right)}\left(\bar{x}_{s-1}\right) \cdots \chi_{R}\left(x_{1}\right),
$$

where $\bar{x}_{i}$ is the image of $x_{i}$ in $R /\left(x_{1}, \ldots, x_{i-1}\right)$. Still further, if $0 \leqq n \leqq s$ then

$$
\chi_{R}\left(x_{1}, \ldots, x_{s}\right)=\chi_{R /\left(x_{1}, \ldots, x_{n}\right)}\left(\bar{x}_{n+1}, \ldots, \bar{x}_{s}\right) \cdot \chi_{R}\left(x_{1}, \ldots, x_{n}\right),
$$

where $\bar{x}_{n+1}, \ldots, \bar{x}_{s}$ are the images of $x_{n+1}, \ldots, x_{s}$ in $R /\left(x_{1}, \ldots, x_{n}\right)$.

Thus we have defined a general multiplicity taking values in a Grothendieck group and have shown that some important properties of this multiplicity depend in no way upon the condition that $R / I$ have finite length. It is easy enough to see how to define the ordinary multiplicity in terms of $\chi_{R}\left(x_{1}, \ldots, x_{s}\right)$. For suppose we are in the case where $I=\left(x_{1}, \ldots, x_{s}\right)$ and $R / I$ has finite length. Then it is known that $K(R / I)$ is isomorphic to $D(R / I)$. In fact, if $M_{1}, \ldots, M_{k}$ are the maximal ideals of $R$ containing $I$, then $M_{1} / I, \ldots, M_{k} / I$ are exactly the prime ideals of $R / I$, and they are all of height zero in $R / I$. So $K(R / I)$ is the free abelian group with basis $\left[R / M_{1}\right], \ldots$, $\left[R / M_{k}\right]$. Now we define a map $l$ from $K(R / I)$ to the integers $Z$ to be the length function; i.e., if $E$ is a module over $R / I$ then $l[E]=L_{R}(E)$. Then the map $l$ is just the unique map from $K(R / I)$ to $Z$ which takes the value 1 on each basis element $\left[R / M_{i}\right], i=1 \ldots, k$.

We shall denote the ordinary multiplicity by $e_{R}\left(x_{1}, \ldots, x_{s} \mid E\right)$ and define it by $e_{R}\left(x_{1}, \ldots, x_{s} \mid E\right)=l_{\chi_{R}}\left(x_{1}, \ldots, x_{s}\right)[E]$. It is easily seen that this definition coincides with that of [1] and [8] and its properties can be derived from our multiplicity $\chi_{R}\left(x_{1}, \ldots, x_{s}\right)$.

It is often the case that properties of the multiplicity map $e_{R}\left(x_{1}, \ldots, x_{s} \mid E\right)$ are stated in terms of length. To prove these properties for $\chi_{R}\left(x_{1}, \ldots, x_{s}\right)$ we must find generalizations which we can prove in terms of Grothendieck groups. The first such property is a generalization of the inequalities $0 \leqq e_{R}\left(x_{1}, \ldots, x_{s} \mid E\right) \leqq L_{R}(E / I E)$. Observe that if $R / I$ has finite length then a module $\tilde{E}$ over $R / I$ goes into the "positive orthant" in $K(R / I)$; i.e., $[\widetilde{E}]=n_{1}\left[R / M_{1}\right]+\cdots+n_{k}\left[R / M_{k}\right]$, where $n_{i} \geqq 0$ for $i=1, \ldots, k$.

1.8. Proposition. For every $R$-module E there exists a module E $\tilde{E}$ over $R / I$ such that $\chi_{R}\left(x_{1}, \ldots, x_{s}\right)[E]=[\tilde{E}]$. Furthermore, $\tilde{E}$ can be taken to be a homomorphic image of $E / I E$.

Proof. Proceed by induction on $s$, using Proposition 1.4 for both $s=1$ and the induction step. 
1.9. Corollary. If $E$ is an $R$-module such that $E=I E$ then $\chi_{R}\left(x_{1}, \ldots, x_{s}\right)[E]=0$.

1.10. Proposition. If $E$ is an $R$-module of finite length then $\chi_{R}\left(x_{1}, \ldots, x_{s}\right)[E]=0$.

Proof. It suffices to prove the proposition for simple modules. This is trivial using Theorem 1.6.

Now we wish to generalize a familiar property of multiplicities found in [1, Proposition 4.4, p. 642]. First observe that if $\mathscr{C}$ and $\mathscr{C}^{\prime}$ are abelian categories and $\mathscr{C}$ is a subcategory of $\mathscr{C}^{\prime}$ then there is a map $\lambda: K^{0}(\mathscr{C}) \rightarrow K^{0}\left(\mathscr{C}^{\prime}\right)$ induced by the inclusion functor $\mathscr{C} \rightarrow \mathscr{C}^{\prime}$. This induced map $\lambda$ is not necessarily monic.

1.11. TheOREM. Let $J=\left(x_{1}, \ldots, x_{i}, \ldots, x_{s}\right), J^{\prime}=\left(x_{1}, \ldots, x_{i}^{\prime}, \ldots, x_{s}\right)$, and $I=$ $\left(x_{1}, \ldots, x_{i} x_{i}^{\prime}, \ldots, x_{s}\right)$. Then $I \subseteq J$ and $I \subseteq J^{\prime}$. Let $\lambda: K(R / J) \rightarrow K(R / I)$ and $\lambda^{\prime}$ : $K\left(R / J^{\prime}\right) \rightarrow K(R / I)$ be the maps induced by the inclusion functors. Then

$$
\chi_{R}\left(x_{1}, \ldots, x_{i} x_{i}^{\prime}, \ldots, x_{s}\right)=\lambda \chi_{R}\left(x_{1}, \ldots, x_{i}, \ldots, x_{s}\right)+\lambda^{\prime} \chi_{R}\left(x_{1}, \ldots, x_{i}^{\prime}, \ldots, x_{s}\right) .
$$

Proof. By Proposition 1.1 we can assume $i=1$. Let $\tau: K\left(R / x_{1} R\right) \rightarrow K\left(R / x_{1} x_{1}^{\prime} R\right)$ and $\tau^{\prime}: K\left(R / x_{1}^{\prime} R\right) \rightarrow K\left(R / x_{1} x_{1}^{\prime} R\right)$ be the maps induced by the inclusion functors. Now the following sequence is exact over $R / x_{1} x_{1}^{\prime} R$ for any $R$-module $E$ :

$$
0 \rightarrow\left(0: x_{1}\right)_{E} \rightarrow\left(0: x_{1} x_{1}^{\prime}\right)_{E} \rightarrow\left(0: x_{1}^{\prime}\right)_{E} \rightarrow E / x_{1} E \rightarrow E / x_{1} x_{1}^{\prime} E \rightarrow E / x_{1}^{\prime} E \rightarrow 0 .
$$

So in $K\left(R / x_{1} x_{1}^{\prime} R\right)$ we have

$$
\left[E / x_{1} x_{1}^{\prime} E\right]-\left[\left(0: x_{1} x_{1}^{\prime}\right)_{E}\right]=\tau\left[E / x_{1} E\right]-\tau\left[\left(0: x_{1}\right)_{E}\right]+\tau^{\prime}\left[E / x_{1}^{\prime} E\right]-\tau^{\prime}\left[\left(0: x_{1}^{\prime}\right)_{E}\right] .
$$

Using Theorem 1.6 it suffices to show the following diagram and a similar one with $\tau^{\prime}$ and $\lambda^{\prime}$ commutes:

$$
\begin{aligned}
& K\left(R / x_{1} R\right) \stackrel{\tau}{\longrightarrow} K\left(R / x_{1} x_{1}^{\prime} R\right)
\end{aligned}
$$

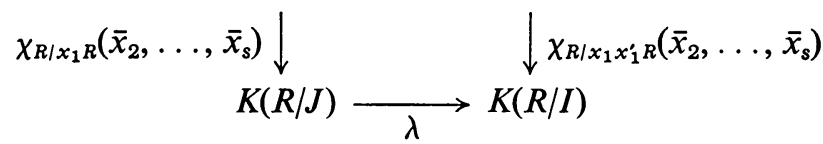

But this is straightforward from the definitions.

1.12. Corollary. Let $n_{1}, \ldots, n_{s}$ be positive integers, let $J=\left(x_{1}, \ldots, x_{s}\right)$, and let $I=\left(x_{1}^{n_{1}}, \ldots, x_{s}^{n_{s}}\right)$. Let $\lambda: K(R / J) \rightarrow K(R / I)$ be the map induced by the inclusion functor. Then $\chi_{R}\left(x_{1}^{n}, \ldots, x_{s}^{n_{s}}\right)=n_{1} \cdots n_{s} \lambda_{R}\left(x_{1}, \ldots, x_{s}\right)$.

We wish to look now at an important property of the multiplicity map. Let $\Phi: R \rightarrow S$ be a ring homomorphism and suppose $S$ is a flat $R$-module. Then we obtain a homomorphism $\Phi^{*}: K(R) \rightarrow K(S)$ induced by the exact functor $\cdot \otimes_{R} S$. If $I$ is an ideal in $R$ then we obtain $\bar{\Phi}: R / I \rightarrow S / \Phi(I)$. If $S / \Phi(I)$ is a flat $R / I$-module we obtain $\bar{\Phi}^{*}: K(R / I) \rightarrow K(S / \Phi(I))$. 
1.13. THEOREM. Under the above hypotheses with $I=\left(x_{1}, \ldots, x_{s}\right)$ the following diagram is commutative:

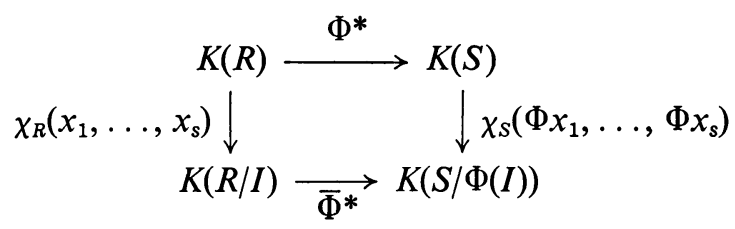

Proof. Use the definitions of the maps together with the fact that $H_{i}\left(E_{x: 1, \ldots, s}\right)$

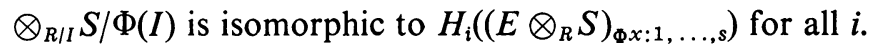

In particular, Theorem 1.13 implies that multiplicity commutes with localization. If $S$ is a multiplicatively closed set in $R$ then $R_{S}$ is a flat $R$-module. We let $T_{S}: K(R) \rightarrow K\left(R_{S}\right)$ denote the induced homomorphism. If $S$ is the complement in $R$ of a prime ideal $P$ we shall also use $T_{P}$ to stand for $T_{S}$. We can then obtain as a corollary to Theorem 1.13 the "Localization Principle" found in [8, Theorem 3, p. 277].

1.14. Corollary. Let $I=\left(x_{1}, \ldots, x_{s}\right)$ and suppose $R / I$ has finite length. Let $M_{1}, \ldots, M_{k}$ be the maximal ideals of $R$ containing $I$ and let $\Phi_{i}: R \rightarrow R_{M_{1}}$ be the natural map. Then

$$
\chi_{R}\left(x_{1}, \ldots, x_{s}\right)[E]=\sum_{i=1}^{k} \chi_{R_{M_{i}}}\left(\Phi_{i} x_{1}, \ldots, \Phi_{i} x_{s}\right)\left[E_{M_{i}}\right] .
$$

Proof. We have $K(R / I)$ is isomorphic to $\oplus_{i=1}^{k} K\left(R_{M_{i}} / I R_{M_{i}}\right)$ and each map $T_{M_{i}}: K(R / I) \rightarrow K\left(R_{M_{i}} / I R_{M_{i}}\right)$ is a projection associated with the direct sum. Thus, using Theorem 1.13,

$$
\begin{aligned}
\chi_{R}\left(x_{1}, \ldots, x_{s}\right)[E] & =\sum_{i=1}^{k} T_{\bar{M}_{i}} \chi_{R}\left(x_{1}, \ldots, x_{s}\right)[E] \\
& =\sum_{i=1}^{k} \chi_{R_{M_{i}}}\left(\Phi_{i} x_{1}, \ldots, \Phi_{i} x_{s}\right)\left[E_{M_{i}}\right] .
\end{aligned}
$$

Now we wish to prove a generalization of the Associative Law for multiplicities. The form of the Associative Law we wish to generalize is that found in [8, Theorem 5 , p. 285]. It can be stated as follows: Let $I=\left(x_{1}, \ldots, x_{s}\right)$ and suppose $R / I$ has finite length. Let $0 \leqq n \leqq s$ and let $P_{1}, \ldots, P_{k}$ be the minimal prime ideals of $\left(x_{1}, \ldots, x_{n}\right)$. Let $\Phi_{i}: R \rightarrow R_{P_{i}}$ and $\psi_{i}: R \rightarrow R / P_{i}$ be the natural maps. Then

$$
e_{R}\left(x_{1}, \ldots, x_{s} \mid E\right)=\sum_{i=1}^{k} e_{R_{P_{i}}}\left(\Phi_{i} x_{1}, \ldots, \Phi_{i} x_{n} \mid E_{P_{i}}\right) e_{R / P_{i}}\left(\psi_{i} x_{n+1}, \ldots, \psi_{i} x_{s} \mid R / P_{i}\right) .
$$

There are several ways the Associative Law can be generalized to Grothendieck groups. We shall state two ways, the first of which is Theorem 1.15. Recall that if $J$ is an ideal in $R$ a set of generators for $K(R / J)$ is $\{[R / P] \mid P$ prime, $P \supseteq J\}$. Now Theorem 1.15 is an easy consequence of Corollary 1.7 . 
1.15. TheOREM. Let $0 \leqq n \leqq s$ and let $\chi_{R}\left(x_{1}, \ldots, x_{s}\right)[E]=\sum_{P} m_{P}[R / P]$ in $K\left(R /\left(x_{1}, \ldots, x_{n}\right)\right)$, where the summation ranges over primes $P$ containing $\left(x_{1}, \ldots, x_{n}\right)$. Then

$$
\chi_{R}\left(x_{1}, \ldots, x_{s}\right)[E]=\sum_{P} m_{P} \lambda_{P} \chi_{R / P}\left(\psi_{P} x_{n+1}, \ldots, \psi_{P} x_{s}\right)[R / P]
$$

where $\psi_{P}: R \rightarrow R / P$ is the natural map and

$$
\lambda_{P}: K\left(R /\left(P, x_{n+1}, \ldots, x_{s}\right)\right) \rightarrow K\left(R /\left(x_{1}, \ldots, x_{s}\right)\right)
$$

is the map induced by the inclusion functor.

If we wish to involve the localization at the primes $P$ in the Associative Law and identify the integers $m_{P}$ we must confine our attention to minimal primes of the ideal $\left(x_{1}, \ldots, x_{n}\right)$. We develop a second generalization from this point of view.

1.16. LemMA. Let $I=\left(x_{1}, \ldots, x_{s}\right)$. If $\rho$ belongs to $Z(R)$ then $\chi_{R}\left(x_{1}, \ldots, x_{s}\right) \rho$ belongs to $Z(R / I)$.

Proof. Proceeding by induction on $s$ we see by Corollary 1.7 that it suffices to consider the case $s=1$. In this case, by definition of $Z(R)$, it suffices to show if $Q$ is a prime in $R$ and height $Q>0$ then $\chi_{R}(x)[R / Q]$ belongs to $Z(R / x R)$. If $x$ is in $Q$ then $\chi_{R}(x)[R / Q]=0$ by Proposition 1.2. So suppose $x$ is not in $Q$. Then $x$ is not a zero-divisor on $Q$ and $\chi_{R}(x)[R / Q]=[R / Q+x R]$. Let $\bar{P}$ be a prime in $R / x R$ which belongs to Supp $(R / Q+x R)$ and let $P$ be the preimage of $\bar{P}$. Then $P \supsetneqq Q$ so height $P$ $>$ height $Q>0$. By the Krull Principal Ideal Theorem, $P$ is not a minimal prime of $x R$. Thus height $\bar{P}>0$, completing the proof.

1.17. Proposition. Let $P_{1}, \ldots, P_{k}$ be the height zero primes of $R$. Then

$$
\chi_{R}\left(x_{1}, \ldots, x_{s}\right)[E]=\sum_{i=1}^{k} L_{R P_{i}}\left(E_{P_{i}}\right) \chi_{R}\left(x_{1}, \ldots, x_{s}\right)\left[R / P_{i}\right]+\rho,
$$

where $\rho$ belongs to $Z(R / I)$.

Proof. In $K(R),[E]=\sum_{i=1}^{k} L_{R_{P_{1}}}\left(E_{P_{i}}\right)\left[R / P_{i}\right]+\rho^{\prime}$, where $\rho^{\prime}$ belongs to $Z(R)$. Now apply $\chi_{R}\left(x_{1}, \ldots, x_{s}\right)$ to both sides and use Lemma 1.16 .

The following corollary is actually a generalization of the Associative Law for the special case $n=0$.

1.18. Corollary. Let $P_{1}, \ldots, P_{k}$ be the primes of $R$ of height zero and let $\psi_{i}: R \rightarrow R / P_{i}$ be the natural map. Then we have

$$
\chi_{R}\left(x_{1}, \ldots, x_{s}\right)[E]=\sum_{i=1}^{k}\left[E_{P_{i}}\right] \otimes \chi_{R / P_{i}}\left(\psi_{i} x_{1}, \ldots, \psi_{i} x_{s}\right)\left[R / P_{i}\right]+\rho,
$$

where $\rho$ belongs to $Z(R / I)$.

Proof. We must note where this tensor product is taking place. Now $\left[E_{P_{i}}\right]$ is an element of $K\left(R_{P_{i}}\right)$ which is infinite cyclic generated by $\left[R_{P_{i}} / P_{i} R_{P_{i}}\right]$. Also

$$
\chi_{R / P_{i}}\left(\psi_{i} x_{1}, \ldots, \psi_{i} x_{s}\right)\left[R / P_{i}\right]
$$


is in $K(R / I)$ and so the tensor product is in $K\left(R_{P_{i}}\right) \otimes_{z} K(R / I)$, which is isomorphic to $K(R / I)$. With these identifications, Corollary 1.18 is a slight modification of Proposition 1.17.

The following theorem is a generalization of the Associative Law for $0 \leqq n \leqq s$. Recall if $R / I$ has finite length then $Z(R / I)=0$.

1.19. TheOREM. Let $I=\left(x_{1}, \ldots, x_{s}\right)$ and suppose $0 \leqq n \leqq s$. Let $P_{1}, \ldots, P_{k}$ be the minimal primes of $\left(x_{1}, \ldots, x_{n}\right)$. Let $\Phi_{i}: R \rightarrow R_{P_{i}}$ and $\psi_{i}: R \rightarrow R / P_{i}$ be the natural maps. Then

$\chi_{R}\left(x_{1}, \ldots, x_{s}\right)[E]=\sum_{i=1}^{k} \chi_{R_{P_{i}}}\left(\Phi_{i} x_{1}, \ldots, \Phi_{i} x_{n}\right)\left[E_{P_{i}}\right] \otimes \chi_{R / P_{i}}\left(\psi_{i} x_{n+1}, \ldots, \psi_{i} x_{s}\right)\left[R / P_{i}\right]+\rho$,

where $\rho$ belongs to $Z(R / I)$.

Proof. Again we must see where this tensor product is taking place. Now $\chi_{R_{P_{i}}}\left(\Phi_{i} x_{1}, \ldots, \Phi_{i} x_{n}\right)\left[E_{P_{i}}\right]$ is an element of $K\left(R_{P_{i}} /\left(x_{1}, \ldots, x_{n}\right) R_{P_{i}}\right)$ which is infinite cyclic. Also $\chi_{R / P_{i}}\left(\psi_{i} x_{n+1}, \ldots, \psi_{i} x_{s}\right)\left[R / P_{i}\right]$ is an element of $K(R / I)$. So the tensor product is $K\left(R_{P_{i}} /\left(x_{1}, \ldots, x_{n}\right) R_{P_{i}}\right) \otimes_{Z} K(R / I)$ which is isomorphic to $K(R / I)$. Now we use respectively Corollary 1.7 , Corollary 1.18 , and Theorem 1.13 . Then

$$
\begin{aligned}
\chi_{R}\left(x_{1}, \ldots, x_{s}\right)[E] & =\chi_{R /\left(x_{1}, \ldots, x_{n}\right)}\left(\bar{x}_{n+1}, \ldots, \bar{x}_{s}\right) \chi_{R}\left(x_{1}, \ldots, x_{n}\right)[E] \\
& =\sum_{i=1}^{k} T_{\bar{P}_{i}} \chi_{R}\left(x_{1}, \ldots, x_{n}\right)[E] \otimes \chi_{R / P_{i}}\left(\psi_{i} x_{n+1}, \ldots, \psi_{i} x_{s}\right)\left[R / P_{i}\right]+\rho \\
& =\sum_{i=1}^{k} \chi_{R_{P_{i}}}\left(\Phi_{i} x_{i}, \ldots, \Phi_{i} x_{n}\right)\left[E_{P_{i}}\right] \otimes \chi_{R / P_{i}}\left(\psi_{i} x_{n+1}, \ldots, \psi_{i} x_{s}\right)\left[R / P_{i}\right]+\rho .
\end{aligned}
$$

Having finished our treatment of the Associative Law we would like to be able to calculate the kernel of $\chi_{R}\left(x_{1}, \ldots, x_{s}\right)$. Although this seems to be difficult in general, we can derive some partial results which, as usual, are most impressive when $R / I$ has finite length. We need some preliminary results. If $R$ is a local ring with maximal ideal $M$ define $\operatorname{dim} R=$ height $M$. Then $\operatorname{dim} R$ is called the Krull dimension of $R$.

1.20. Proposition. Let $R$ be a local ring with maximal ideal $M$ and let $x_{1}, \ldots, x_{s}$ be elements of $M$. Let $I=\left(x_{1}, \ldots, x_{s}\right)$ and suppose $R / I$ has finite length. If $P$ is prime in $R$ then $\chi_{R}\left(x_{1}, \ldots, x_{s}\right)[R / P] \neq 0$ if, and only if, $s=\operatorname{dim} R / P$.

Note. Since $R / I$ has finite length, $s \geqq \operatorname{dim} R \geqq \operatorname{dim} R / P$. If $s=\operatorname{dim} R / P$ then height $P=0$.

Proof. Let $\psi: R \rightarrow R / P$ be the natural map. Suppose first $s=\operatorname{dim} R / P$. Then $s=\operatorname{dim} R$ and $x_{1}, \ldots, x_{s}$ is a system of parameters in $R$. We show $\chi_{R}\left(x_{1}, \ldots, x_{s}\right)$ $\cdot[R / P] \neq 0$ by induction on $s$. The case $s=0$ is trivial. So suppose $s>0$ and make the induction hypothesis. Since $\chi_{R}\left(x_{1}, \ldots, x_{s}\right)[R / P]=\chi_{R / P}\left(\psi x_{1}, \ldots, \psi x_{s}\right)[R / P]$, we can assume $R$ is an integral domain of Krull dimension $s$ and we must show $\chi_{R}\left(x_{1}, \ldots, x_{s}\right)[R] \neq 0$. But $x_{1}$ is not a zero-divisor on $R$ and so $\chi_{R}\left(x_{1}, \ldots, x_{s}\right)[R]$ 
$=\chi_{R / x_{1} R}\left(\bar{x}_{2}, \ldots, \bar{x}_{s}\right)\left[R / x_{1} R\right]$ and, by [6, Corollary 2, p. 65], $R / x_{1} R$ is a local ring of Krull dimension $s-1$. By the induction hypothesis, if $Q_{1}, \ldots, Q_{t}$ are the primes of $\bar{R}=R / x_{1} R$ such that $\operatorname{dim} \bar{R} / Q_{i}=s-1$ then $\chi_{\bar{R}}\left(\bar{x}_{2}, \ldots, \bar{x}_{s}\right)\left[\bar{R} / Q_{i}\right] \neq 0$ in $K(R / I)$. Since $K(R / I)$ is free and there exists at least one such $Q_{i}, \chi_{\bar{R}}\left(\bar{x}_{2}, \ldots, \bar{x}_{s}\right)[\bar{R}] \neq 0$ completing the induction.

Now suppose $s>\operatorname{dim} R / P$. We show $\chi_{R}\left(x_{1}, \ldots, x_{s}\right)[R / P]=0$ by induction on $s$. If $s=1$ then $\operatorname{dim} R / P=0$ and $x_{1}^{n} \cdot R / P=0$ for some $n$. So $\chi_{R}\left(x_{1}\right)[R / P]=0$ by Proposition 1.2. So suppose $s>1$ and make the induction hypothesis. If $x_{1}$ is in $P$ then $\chi_{R}\left(x_{1}, \ldots, x_{s}\right)[R / P]=0$ by Proposition 1.2. If $x_{1}$ is not in $P$ then $\chi_{R}\left(x_{1}, \ldots, x_{s}\right)[R / P]$ $=\chi_{R / x_{1} R}\left(\bar{x}_{2}, \ldots, \bar{x}_{s}\right)\left[R / P+x_{1} R\right]$ by Theorem 1.6. Now in $K\left(R / x_{1} R\right)$ we have $\left[R / P+x_{1} R\right]=\sum n_{Q}[R / Q]$, where $Q$ ranges over primes of $R$ containing $P+x_{1} R$. Thus $\operatorname{dim} R / Q<\operatorname{dim} R / P \leqq s-1$ and, by the induction hypothesis,

$$
\chi_{R / x_{1} R}\left(x_{2}, \ldots, x_{s}\right)[R / Q]=0 .
$$

Thus $\chi_{R}\left(x_{1}, \ldots, x_{s}\right)[R / P]=0$, completing the proof.

Now we return to the case where $R$ is not necessarily a local ring. If $I$ is an ideal of $R$, not necessarily prime, then there are several ways to define height $I$. For our purposes we want the following: height $I$ is the maximum of the heights of the minimal primes containing $I$. By the Krull Principal Ideal Theorem, if $I=\left(x_{1}, \ldots, x_{s}\right)$ then height $I \leqq s$.

1.21. Corollary. If $I=\left(x_{1}, \ldots, x_{s}\right)$ and $s>$ height $I$ then for all $\rho$ in $K(R)$, $\chi_{R}\left(x_{1}, \ldots, x_{s}\right) \rho$ belongs to $Z(R / I)$.

Proof. By Lemma 1.16, it suffices to show that if $P$ is a height zero prime in $R$ then $\chi_{R}\left(x_{1}, \ldots, x_{s}\right)[R / P]$ is in $Z(R / I)$. Now $Z(R / I)=\bigcap \operatorname{ker} T_{\bar{Q}}$, where $\bar{Q}$ ranges over the height zero primes of $R / I$. So it suffices to show $T_{\bar{Q} \chi_{R}}\left(x_{1}, \ldots, x_{s}\right)[R / P]=0$ for all such $\bar{Q}$. By Theorem 1.13, it suffices to show $\chi_{R_{Q}}\left(\Phi x_{1}, \ldots, \Phi x_{s}\right) T_{Q}[R / P]=0$ for all minimal primes $Q$ of $I$ where $\Phi: R \rightarrow R_{Q}$ is the natural map. By hypothesis, $\operatorname{dim} R_{Q}=$ height $Q<s$, and so, by Proposition $1.20, \chi_{R_{Q}}\left(\Phi x_{1}, \ldots, \Phi x_{s}\right) T_{Q}[R / P]=0$.

In the case where $R / I$ has finite length and $s>$ height $I$ Corollary 1.21 tells the whole story. For then $Z(R / I)=0$ and so $\chi_{R}\left(x_{1}, \ldots, x_{s}\right)$ is the zero map. The more interesting case is when $s=$ height $I$.

1.22. Corollary. Let $I=\left(x_{1}, \ldots, x_{s}\right)$ and let $Q$ be a minimal prime of $I$ of height s. If $P$ is a prime in $R$ such that $P \subseteq Q$ and $\operatorname{dim} R_{Q} / P R_{Q}=s$ then $\chi_{R}\left(x_{1}, \ldots, x_{s}\right)[R / P]$ is not in $Z(R / I)$.

Proof. This follows from Proposition 1.20 by localizing at $Q$.

1.23. Lemma. Let $I=\left(x_{1}, \ldots, x_{s}\right)$ and let $P$ be a prime of $R$. If there is no minimal prime $Q$ of $I$ of height $s$ such that $\operatorname{dim} R_{Q} / P R_{Q}=s$ then $\chi_{R}\left(x_{1}, \ldots, x_{s}\right)[R / P]$ belongs to $Z(R / I)$. 
Proof. It suffices to show if $Q$ is a minimal prime of $I$ in $R$ and $\Phi: R \rightarrow R_{Q}$ is the natural map then $\chi_{R_{Q}}\left(\Phi x_{1}, \ldots, \Phi x_{s}\right) T_{Q}[R / P]=0$. If $P \nsubseteq Q$ then $T_{Q}[R / P]=0$. If $P \subseteq Q$ then, by hypothesis, $\operatorname{dim} R_{Q} / P R_{Q}<s$, and Proposition 1.20 covers this case.

1.24. Corollary. Let $I=\left(x_{1}, \ldots, x_{s}\right)$ and let $P$ be $a$ prime in $R$. Then $\chi_{R}\left(x_{1}, \ldots, x_{s}\right)[R / P]$ does not belong to $Z(R / I)$ if, and only if, there exists a minimal prime $Q$ of I of height s such that $P \subseteq Q$ and $\operatorname{dim} R_{Q} / P R_{Q}=s$.

Now let $Q_{1}, \ldots, Q_{t}$ be the minimal primes of $I$ of height $s$ and let $P_{1}, \ldots, P_{k}$ be the primes of $R$ which satisfy the condition that $P_{i}$ is contained in some $Q_{j}$ and $\operatorname{dim} R_{Q_{j}} / P_{i} R_{Q_{j}}=s$. Let $\rho=n_{1}\left[R / P_{1}\right]+\cdots+n_{k}\left[R / P_{k}\right]+\rho^{\prime}$ be an element of $K(R)$ where $\rho^{\prime}$ belongs to $Z(R)$ and suppose $\chi_{R}\left(x_{1}, \ldots, x_{s}\right) \rho$ belongs to $Z(R / I)$. Now let $Q_{1}, \ldots$, $Q_{t}, Q_{t+1}, \ldots, Q_{j}$ be all the minimal primes of $I$. Then for each $i=1, \ldots, k$,

$$
\chi_{R}\left(x_{1}, \ldots, x_{s}\right)\left[R / P_{i}\right]=m_{i 1}\left[R / Q_{1}\right]+\cdots+m_{i j}\left[R / Q_{j}\right]+\rho_{i},
$$

where $\rho_{i}$ is in $Z(R / I)$ and $m_{i l} \geqq 0$ for all $l$, not all $m_{i l}=0$. Then

is in $Z(R / I)$. So

$$
n_{1} \sum_{l=1}^{j} m_{1 l}\left[R / Q_{l}\right]+\cdots+n_{k} \sum_{l=1}^{j} m_{k l}\left[R / Q_{l}\right]
$$

$$
\left(n_{1} m_{11}+\cdots+n_{k} m_{k 1}\right)\left[R / Q_{1}\right]+\cdots+\left(n_{1} m_{1 j}+\cdots+n_{k} m_{k j}\right)\left[R / Q_{j}\right]=0 .
$$

Thus

$$
\begin{gathered}
n_{1} m_{11}+\cdots+n_{k} m_{k 1}=0 \\
\vdots \\
n_{1} m_{1 j}+\cdots+n_{k} m_{k j}=0
\end{gathered}
$$

Now in each column there exists at least one $m_{i l}>0$. This shows that if $n_{i}>0$ for some $i$ then there exists an $i^{\prime}$ such that $n_{i^{\prime}}<0$. But if $\rho=[E]$ for some module $E$ then we can choose $n_{i} \geqq 0$ for all $i$. This proves the following theorem.

1.25. TheOREM. Let $I=\left(x_{1}, \ldots, x_{s}\right)$ and suppose $s=$ height $I$. Let $Q_{1}, \ldots, Q_{t}$ be the minimal primes of $I$ of height s. Let $P_{1}, \ldots, P_{k}$ be the primes of $R$ which satisfy the condition that each $P_{i}$ is contained in some $Q_{j}$ and $\operatorname{dim} R_{Q_{j}} / P_{i} R_{Q_{j}}=s$. If $E$ is an $R$-module then $\chi_{R}\left(x_{1}, \ldots, x_{s}\right)[E]$ belongs to $Z(R / I)$ if, and only if, $E_{P_{i}}=0$ for $i=1, \ldots, k$.

We wish finally to turn our attention to the Extension Formula. This formula shows how the multiplicity changes under integral extension. The theorem we shall obtain is found in [8, Theorem 6, p. 287]. Let $R$ be a subring of $R^{\prime}$ and suppose $R^{\prime}$ is a finitely generated $R$-module. If $x_{1}, \ldots, x_{s}$ are elements of $R$ such that $R^{\prime} / x_{1} R^{\prime}+\cdots+x_{s} R^{\prime}$ has finite length and $E^{\prime}$ is an $R^{\prime}$-module then

$$
e_{R}\left(x_{1}, \ldots, x_{s} \mid E^{\prime}\right)=\sum_{M^{\prime}} L_{R / M^{\prime} \cap R}\left(R^{\prime} / M^{\prime}\right) e_{R_{M^{\prime}}^{\prime}}\left(\Phi_{M^{\prime}} x_{1}, \ldots, \Phi_{M^{\prime}} x_{s} \mid E_{M^{\prime}}^{\prime}\right),
$$

where $M^{\prime}$ ranges over the maximal ideals of $R^{\prime}$ containing $x_{1} R^{\prime}+\cdots+x_{s} R^{\prime}$ and $\Phi_{M^{\prime}}: R^{\prime} \rightarrow R_{M^{\prime}}^{\prime}$ is the natural map. 
Naturally we wish to generalize the Extension Formula to our case. It is somewhat surprising that the Extension Formula receives a much more simple formulation with Grothendieck groups. Recall if $\Phi: R \rightarrow R^{\prime}$ is a ring homomorphism then every finitely generated $R^{\prime}$-module is a finitely generated $R$-module. So we obtain a homomorphism $\Phi_{*}: K\left(R^{\prime}\right) \rightarrow K(R)$ induced by the inclusion functor. If $I$ is an ideal in $R$ then $\bar{\Phi}: R / I \rightarrow R^{\prime} / \Phi(I)$ is a ring homomorphism and we obtain

$$
\bar{\Phi}_{*}: K\left(R^{\prime} / \Phi(I)\right) \rightarrow K(R / I) .
$$

1.26. THEOREM( $\left.{ }^{2}\right)$. Let $\Phi: R \rightarrow R^{\prime}$ be a ring homomorphism and let $I=\left(x_{1}, \ldots, x_{s}\right)$ be an ideal of $R$. Then the following diagram commutes:

$$
\begin{gathered}
K\left(R^{\prime}\right) \stackrel{\Phi_{*}}{\longrightarrow} K(R) \\
\chi_{R^{\prime}}\left(\Phi x_{1}, \ldots, \Phi x_{s}\right) \downarrow_{K\left(R^{\prime} / \Phi(I)\right) \stackrel{\Phi_{*}}{\longrightarrow} K(R / I)}^{\downarrow} \chi_{R}\left(x_{1}, \ldots, x_{s}\right)
\end{gathered}
$$

To obtain the Extension Formula as stated above we apply Theorem 1.26 to the special case where $R$ is a subring of $R^{\prime}$ and $\Phi: R \rightarrow R^{\prime}$ is the inclusion. We need some lemmas. The proof of the following lemma is found in $[8$, Proof of Theorem 6, p. 287].

1.27. LemMa. If $R$ is a subring of $R^{\prime}, x_{1}, \ldots, x_{s}$ are elements of $R$, and $R^{\prime} / x_{1} R^{\prime}$ $+\cdots+x_{s} R^{\prime}$ has finite length then $R / x_{1} R+\cdots+x_{s} R$ has finite length. If $M^{\prime}$ is $a$ maximal ideal of $R^{\prime}$ then $M=M^{\prime} \cap R$ is a maximal ideal of $R$ and $L_{R / M}\left(R^{\prime} / M^{\prime}\right)$ $=L_{R_{M}}\left(\left(R^{\prime} / M^{\prime}\right)_{M}\right)$.

1.28. Lemma. If $R^{\prime \prime}=R^{\prime} / x_{1} R^{\prime}+\cdots+x_{s} R^{\prime}$ has finite length and $E$ is an $R^{\prime \prime}$-module then

$$
\bar{\Phi}_{*}[E]=\sum_{M^{\prime}} L_{R^{\prime}}\left(E_{M^{\prime}}\right) L_{R / M^{\prime} \cap R}\left(R^{\prime} / M^{\prime}\right)\left[R / M^{\prime} \cap R\right]
$$

in $K\left(R / x_{1} R+\cdots+x_{s} R\right)$, where $M^{\prime}$ ranges over the maximal ideals of $R^{\prime}$ containing $x_{1} R^{\prime}+\cdots+x_{s} R^{\prime}$.

Proof. Since $R / x_{1} R+\cdots+x_{s} R$ has finite length,

$$
\bar{\Phi}_{*}\left[R^{\prime} / M^{\prime}\right]=\sum_{M} L_{R_{M}}\left(\left(R^{\prime} / M^{\prime}\right)_{M}\right)[R / M]
$$

where $M$ ranges over the maximal ideals of $R$ containing $x_{1} R+\cdots+x_{s} R$. Using Lemma 1.27,

But

$$
\bar{\Phi}_{*}\left[R^{\prime} / M^{\prime}\right]=L_{R / M^{\prime} \cap R}\left(R^{\prime} / M^{\prime}\right)\left[R / M^{\prime} \cap R\right]
$$

$$
\bar{\Phi}_{*}[E]=\sum_{M^{\prime}} L_{R_{M^{\prime}}^{\prime}}\left(E_{M^{\prime}}\right) \bar{\Phi}_{*}\left[R^{\prime} / M^{\prime}\right]=\sum_{M^{\prime}} L_{R^{\prime}}\left(E_{M^{\prime}}\right) L_{R / M^{\prime} \cap R}\left(R^{\prime} / M^{\prime}\right)\left[R / M^{\prime} \cap R\right] .
$$

$\left.{ }^{2}\right)$ I would like to thank the referee for pointing out the appropriate generality for this theorem. 
1.29. THEOREM. Let $R$ be a subring of $R^{\prime}$ and suppose $R^{\prime}$ is a finitely generated $R$-module. If $x_{1}, \ldots, x_{s}$ are elements of $R$ and $R^{\prime} / x_{1} R^{\prime}+\cdots+x_{s} R^{\prime}$ has finite length then

$$
e_{R}\left(x_{1}, \ldots, x_{s} \mid E^{\prime}\right)=\sum_{M^{\prime}} L_{R / M^{\prime} \cap R}\left(R^{\prime} / M^{\prime}\right) e_{R_{M^{\prime}}^{\prime}}\left(\Phi_{M^{\prime}} x_{1}, \ldots, \Phi_{M^{\prime}} x_{s} \mid E_{M^{\prime}}^{\prime}\right),
$$

where $M^{\prime}$ ranges over the maximal ideals of $R^{\prime}$ containing $x_{1} R^{\prime}+\cdots+x_{s} R^{\prime}$ and $\Phi_{M^{\prime}}: R^{\prime} \rightarrow R_{M^{\prime}}^{\prime}$ is the natural map.

Proof. Let $l$ denote the length homomorphism. We use respectively Theorem 1.26, Corollary 1.14, and Lemma 1.28.

$$
\begin{aligned}
& e_{R}\left(x_{1}, \ldots, x_{s} \mid E^{\prime}\right)=l_{\chi_{R}}\left(x_{1}, \ldots, x_{s}\right) \Phi_{*}\left[E^{\prime}\right] \\
& =l \bar{\Phi}_{* \chi_{R^{\prime}}}\left(x_{1}, \ldots, x_{s}\right)\left[E^{\prime}\right] \\
& =\sum_{M^{\prime}} l \bar{\Phi}_{*} \chi_{R_{M^{\prime}}^{\prime}}\left(\Phi_{M^{\prime}} x_{1}, \ldots, \Phi_{M^{\prime}} x_{s}\right)\left[E_{M^{\prime}}^{\prime}\right]
\end{aligned}
$$

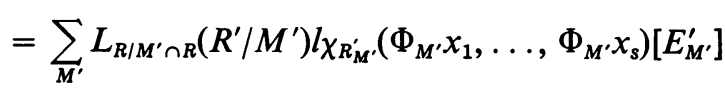

$$
\begin{aligned}
& =\sum_{M^{\prime}} L_{R / M^{\prime} \cap R}\left(R^{\prime} / M^{\prime}\right) e_{R_{M^{\prime}}^{\prime}}\left(\Phi_{M^{\prime}} x_{1}, \ldots, \Phi_{M^{\prime}} x_{s}\right)\left[E_{M^{\prime}}^{\prime}\right] \text {, }
\end{aligned}
$$

using in the last step that $L_{R / M^{\prime} \cap R}\left(R / M^{\prime} \cap R\right)=1$.

2. Hilbert functions. In this section we define the Hilbert function of a module with respect to an ideal in terms of Grothendieck groups. We prove the Hilbert function is a polynomial for large values of $n$ which we call the Samuel polynomial. Then we prove a fundamental relation between the Samuel polynomial and the multiplicity map.

2.1. Definition. Let $I$ be an ideal in $R$ and let $E$ be an $R$-module. Define $H_{I}(n, E)=\left[I^{n} E / I^{n+1} E\right]$ in $K(R / I)$. Then $H_{I}(n, E)$ is called the Hilbert function of $E$ with respect to $I$.

2.2. Definition. Suppose $\alpha_{0}, \alpha_{1}, \ldots, \alpha_{s}$ are elements of $K(R)$ and let

$$
P(n)=\sum_{k=0}^{s-1}\left(\begin{array}{c}
n+k \\
k
\end{array}\right) \alpha_{k} .
$$

Then $P(n)$ is called a Samuel polynomial with coefficients in $K(R)$. The degree of $P(n)$ is the largest integer $t$ such that $\alpha_{t} \neq 0$.

2.3. Proposition. Let $P(n)=\sum_{k=0}^{s} C_{n+k, k} \alpha_{k}$ and $Q(n)=\sum_{k=0}^{s} C_{n+k, k} \beta_{k}$ be two Samuel polynomials with coefficients in $K(R)$. If $P(n)=Q(n)$ for all sufficiently large values of $n$ then $\alpha_{k}=\beta_{k}$ for $k=0,1, \ldots, s$.

Now let $I=\left(x_{1}, \ldots, x_{s}\right)$ and let $E$ be an $R$-module. Let $M=\bigoplus_{n=0}^{\infty} M_{n}$, where $M_{n}=I^{n} E / I^{n+1} E$. Then $M$ is a graded module over $R / I\left[X_{1}, \ldots, X_{s}\right]$ with the operation:

$$
X_{i}\left(e+I^{n+1} E\right)=x_{i} e+I^{n+2} E .
$$


So we have a graded module $M$ which is finitely generated over $R / I\left[X_{1}, \ldots, X_{S}\right]$ and each graded component $M_{n}$ is finitely generated as a module over $R / I$. But this is sufficient to imply that the function $P(n)=\left[M_{n}\right]$ in $K(R / I)$ is a Samuel polynomial of degree at most $s-1$ for all sufficiently large $n$. The proof is by induction on $s$. The case $s=0$ is implied by the fact that $M$ is a finitely generated graded module over $R / I$. The induction step is implied by the exact sequence:

$$
0 \longrightarrow\left(0: X_{s}\right)_{n-1} \longrightarrow M_{n-1} \stackrel{X_{s}}{\longrightarrow} M_{n} \longrightarrow\left(M / X_{s} M\right)_{n} \longrightarrow 0 .
$$

Thus we have the following theorem.

2.4. TheOREM. Let $I=\left(x_{1}, \ldots, x_{s}\right)$. If $E$ is an $R$-module then there exists a Samuel polynomial $P_{I}(n, E)$ with coefficients in $K(R / I)$ and degree at most $s-1$ such that $H_{I}(n, E)=P_{I}(n, E)$ for all sufficiently large $n$.

Actually we find it convenient to deal not only with the Hilbert function but also with another function which we now define. Let

$$
H_{I}^{*}(n, E)=\sum_{k=0}^{n} H_{I}(k, E)
$$

Then $H_{I}^{*}(n, E)$ is called the cumulative Hilbert function of $E$ with respect to $I$ and takes values in $K(R / I)$. But now let $\mathscr{A}_{I}$ be the category of $R$-modules $E$ such that $I^{k} E=0$ for some $k$ (depending on $E$ ). Then it is easy to see that $K^{0}\left(\mathscr{A}_{I}\right)$ is isomorphic to $K(R / I)$. Using the filtration $E \supseteq I E \supseteq \cdots \supseteq I^{n+1} E$ we obtain

$$
\left[E / I^{n+1} E\right]=\sum_{k=0}^{n}\left[I^{k} E / I^{k+1} E\right]
$$

in $K(R / I)$. So we see that $H_{I}^{*}(n, E)=\left[E / I^{n+1} E\right]$ for all $n$.

2.5. Corollary. Let $I=\left(x_{1}, \ldots, x_{s}\right)$. If $E$ is an $R$-module then there exists a Samuel polynomial $P_{I}^{*}(n, E)$ with coefficients in $K(R / I)$ and degree at most s such that $H_{I}^{*}(n, E)=P_{I}^{*}(n, E)$ for all sufficiently large values of $n$.

The next theorem establishes the relationships between our general multiplicity and Hilbert functions. First suppose $P(n)$ is any Samuel polynomial with coefficients in a Grothendieck group. Define $\Delta P(n)=P(n)-P(n-1)$. We note that $\Delta P(n)$ is a Samuel polynomial with degree one less than the degree of $P(n)$. In fact, if

$$
P(n)=\sum_{k=0}^{s}\left(\begin{array}{c}
n+k \\
k
\end{array}\right) \alpha_{k}
$$

then

$$
\Delta P(n)=\sum_{k=1}^{n}\left(\begin{array}{c}
n+k-1 \\
k-1
\end{array}\right) \alpha_{k}=\sum_{i=0}^{s-1}\left(\begin{array}{c}
n+i \\
i
\end{array}\right) \alpha_{i+1} .
$$


For the Samuel polynomials defined in Theorem 2.4 and Corollary 2.5 we see that $\Delta P_{I}^{*}(n, E)=P_{I}(n, E)$. More generally we see that

$$
\Delta^{k} P(n)=\sum_{i=0}^{k}(-1)^{i}\left(\begin{array}{l}
k \\
i
\end{array}\right) P(n-i) .
$$

2.6. THEOREM. Let $I=\left(x_{1}, \ldots, x_{s}\right)$. If $E$ is an $R$-module then $\chi_{R}\left(x_{1}, \ldots, x_{s}\right)[E]$ $=\Delta^{s} P_{I}^{*}(n, E)$.

Proof. Let $C=E_{x: 1, \ldots, s}$ be the Koszul complex of $E$ generated by $x_{1}, \ldots, x_{s}$. For each $k$ define the following subcomplex of $C$ :

$$
C^{(k)}: 0 \rightarrow I^{k} C_{s} \rightarrow I^{k+1} C_{s-1} \rightarrow \cdots \rightarrow I^{k+s} C_{0} \rightarrow 0,
$$

with the differentiation of $C^{(k)}$ induced by that of $C$. Then we obtain the exact sequence of complexes:

$$
0 \rightarrow C^{(k)} \rightarrow C \rightarrow C / C^{(k)} \rightarrow 0 .
$$

By [4] we have $H\left(C^{(k)}\right)=0$ for all $k$ sufficiently large. Then $H_{i}\left(C / C^{(k)}\right)$ is isomorphic to $H_{i}(C)$ for all $i$ and all $k$ sufficiently large. Thus in $K^{0}\left(\mathscr{A}_{I}\right)$ we have

$$
\begin{aligned}
\chi_{R}\left(x_{1}, \ldots, x_{s}\right)[E] & =\sum_{i=0}^{s}(-1)^{i}\left[H_{i}(C)\right]=\sum_{i=0}^{s}(-1)^{i}\left[H_{i}\left(C / C^{(k)}\right)\right] \\
& =\sum_{i=0}^{s}(-1)^{i}\left[\left(C / C^{(k)}\right)_{i}\right]=\sum_{i=0}^{s}(-1)^{i}\left[C_{i} / I^{k+s-i} C_{i}\right] \\
& =\sum_{i=0}^{s}(-1)^{i}\left[C_{i} / I^{n-i+1} C_{i}\right] \quad(\text { let } n=k+s-1) \\
& =\sum_{i=0}^{s}(-1)^{i}\left(\begin{array}{l}
s \\
i
\end{array}\right)\left[E / I^{n-i+1} E\right] \\
& =\Delta^{s}\left[E / I^{n+1} E\right]=\Delta^{s} P_{I}^{*}(n, E) .
\end{aligned}
$$

Incidentally, Theorem 2.6 can be used to prove Corollary 2.5. For if $\Delta P(n)$ is a Samuel polynomial of degree at most $k$ then $P(n)$ is a Samuel polynomial of degree at most $k+1$.

Theorem 2.6 shows that the multiplicity map $\chi_{R}\left(x_{1}, \ldots, x_{s}\right)$ depends only on the ideal generated by $x_{1}, \ldots, x_{s}$ and $s$, the number of elements used to generate the ideal.

2.7. Corollary. Let $x_{1}, \ldots, x_{s}$ and $y_{1}, \ldots, y_{s}$ be elements of $R$ such that $\left(x_{1}, \ldots, x_{s}\right)=I=\left(y_{1}, \ldots, y_{s}\right)$. Then $\chi_{R}\left(x_{1}, \ldots, x_{s}\right)=\chi_{R}\left(y_{1}, \ldots, y_{s}\right)$. Furthermore, if $0 \leqq n \leqq s$ and $0 \leqq m \leqq s$ then

$\chi_{R /\left(x_{1}, \ldots, x_{n}\right)}\left(\bar{x}_{n+1}, \ldots, \bar{x}_{s}\right) \chi_{R}\left(x_{1}, \ldots, x_{n}\right)=\chi_{R /\left(y_{1}, \ldots, y_{m}\right)}\left(\bar{y}_{m+1}, \ldots, \bar{y}_{s}\right) \chi_{R}\left(y_{1}, \ldots, y_{m}\right)$.

2.8. Corollary. Let $x_{1}, \ldots, x_{s}$ and $y_{1}, \ldots, y_{s+1}$ be elements of $R$ such that $\left(x_{1}, \ldots, x_{s}\right)=I=\left(y_{1}, \ldots, y_{s+1}\right)$. Then $\chi_{R}\left(y_{1}, \ldots, y_{s+1}\right)=0$. 
We introduce the notation $\mu(I)$ to denote the minimal cardinality of a set of generators of the ideal $I$.

2.9. Corollary. Let $I=\left(x_{1}, \ldots, x_{s}\right)$. If for some $R$-module $E, \chi_{R}\left(x_{1}, \ldots, x_{s}\right)[E]$ $\neq 0$, then $s=\mu(I)$.

We note that we have shown above degree $P_{I}^{*}(n, E) \leqq \mu(I)$ for an $R$-module $E$.

Now we wish to establish a result for the Samuel polynomial analogous to Theorem 1.6 for multiplicities.

2.10. Lemma. Let $I$ be an ideal and let $x$ belong to $I$. Then

$$
H_{I}(n, E)=H_{I / x R}^{*}(n, E / x E)-\left[\left(I^{n+1} E: x\right) / I^{n} E\right] .
$$

Proof. This follows from the exact sequence:

$$
0 \longrightarrow\left(I^{n+1} E: x\right) / I^{n} E \longrightarrow E / I^{n} E \stackrel{x}{\longrightarrow} E / I^{n+1} E \longrightarrow E / I^{n+1} E+x E \longrightarrow 0 .
$$

Now suppose $I=\left(x_{1}, \ldots, x_{s}\right)$. There exists a Samuel polynomial $Q(n)$ such that $Q(n)=\left[\left(I^{n+1} E: x_{1}\right) / I^{n} E\right]$ for sufficiently large values of $n$. Thus

$$
\Delta P_{I}^{*}(n, E)=P_{I / x_{1} R}^{*}\left(n, E / x_{1} E\right)-Q(n)
$$

and so

Thus

$$
\Delta^{s} P_{I}^{*}(n, E)=\Delta^{s-1} P_{I / x_{1} R}^{*}\left(n, E / x_{1} E\right)-\Delta^{s-1} Q(n)
$$

$$
\chi_{R}\left(x_{1}, \ldots, x_{s}\right)[E]=\chi_{R / x_{1} R}\left(\bar{x}_{2}, \ldots, \bar{x}_{s}\right)\left[E / x_{1} E\right]-\Delta^{s-1} Q(n)
$$

which implies

$$
\Delta^{s-1} Q(n)=\chi_{R \mid x_{1} R}\left(\bar{x}_{2}, \ldots, \bar{x}_{s}\right)\left[\left(0: x_{1}\right)_{E}\right]=\Delta^{s-1} P_{I \mid x_{1} R}^{*}\left(n,\left(0: x_{1}\right)_{E}\right) .
$$

Thus we have shown the following:

2.11. Proposition. If $I=\left(x_{1}, \ldots, x_{s}\right)$ then

$$
\Delta P_{I}^{*}(n, E)=P_{I / x_{1} R}^{*}\left(n, E / x_{1} E\right)-P_{I \mid x_{1} R}^{*}\left(n,\left(0: x_{1}\right)_{E}\right)+P(n),
$$

where $P(n)$ is a Samuel polynomial of degree $<s-1$.

We wish to conclude this section with some results concerning the degree of the Samuel polynomial.

2.12. Proposition. Let $S$ be a multiplicatively closed set in $R$. If $P_{i}^{*}(n, E)=$ $\sum_{k=0}^{m} C_{n+k, k} \alpha_{k}$ then $P_{I R_{S}}^{*}\left(n, E_{S}\right)=\sum_{k=0}^{m} C_{n+k, k} T_{S}\left(\alpha_{k}\right)$. In particular,

$$
\text { degree } P_{I R_{S}}^{*}\left(n, E_{S}\right) \leqq \text { degree } P_{I}^{*}(n, E) \text {. }
$$

Now we make the observation that, by Theorem $2.6, \chi_{R}\left(x_{1}, \ldots, x_{s}\right)[E] \neq 0$ implies degree $P_{I}^{*}(n, E)=s$. In particular, if $R$ is a local ring of Krull dimension $s$ and $I$ is primary for the maximal ideal then, by Proposition 1.20 , degree $P_{I}^{*}(n, R)=s$. 
Return now to the general case, $R$ a ring, $I$ an ideal. Suppose $P_{I}^{*}(n, E)$ $=\sum_{k=0}^{m} C_{n+k, k} \alpha_{k}$. Define $s$ to be the reduced degree of $P_{I}^{*}(n, E)$ if $\alpha_{s}$ is not in $Z(R / I)$ but $\alpha_{k}$ is in $Z(R / I)$ for $k>s$. Obviously reduced degree $P_{I}^{*}(n, E) \leqq$ degree $P_{I}^{*}(n, E)$ and equality holds if $Z(R / I)=0$.

2.13. Proposition. Reduced degree $P_{I}^{*}(n, R)=$ height $I$.

Proof. Let $Q_{1}, \ldots, Q_{j}$ be the minimal primes of $I$ and let $\bar{Q}_{i}$ be the image of $Q_{i}$ in $R / I$. Suppose $P_{I}^{*}(n, R)=\sum_{k=0}^{m} C_{n+k, k} \alpha_{k}$ and has reduced degree $s$. Since $Z(R / I)$ $=\bigcap_{i=1}^{j} \operatorname{ker} T_{\bar{Q}_{i}}$, there exists an $i$ such that $T_{\bar{Q}_{i}}\left(\alpha_{s}\right) \neq 0$. So reduced degree

$$
\begin{aligned}
P_{I}^{*}(n, R) & =\max _{i}\left\{\text { degree } P_{I R_{Q}}^{*}\left(n, R_{Q_{i}}\right)\right\} \\
& =\max _{i}\left\{\operatorname{dim} R_{Q_{i}}\right\}=\max _{i}\left\{\text { height } Q_{i}\right\}=\text { height } I .
\end{aligned}
$$

3. Miscellaneous results. In this section we prove two results of a somewhat special nature. Recall if $E$ is an $R$-module a sequence $x_{1}, \ldots, x_{s}$ of elements of $R$ is an $E$-sequence of length $s$ if $x_{i}$ is not a zero-divisor on $E /\left(x_{1}, \ldots, x_{i-1}\right) E$ for $i=1, \ldots, s$.

3.1. Proposition. If $I$ is an ideal which is generated by an E-sequence of length $s$ then $H_{I}^{*}(n, E)=C_{n+s, s}[E / I E]$ for all $n$.

Proof. We proceed by induction on $s$. The case $s=1$ follows from the exact sequence:

$$
0 \rightarrow x E+\left(0: x^{n}\right)_{E} \rightarrow E \rightarrow x^{n} E / x^{n+1} E \rightarrow 0 .
$$

So suppose $s>1$ and make the induction hypothesis. Suppose $I=\left(y_{1}, \ldots, y_{s}\right)$ where $y_{1}, \ldots, y_{s}$ is an $E$-sequence. By [5] there exists an $E$-sequence $x_{1}, \ldots, x_{s}$ such that $I=\left(x_{1}, \ldots, x_{s}\right)$ and any permutation of $x_{1}, \ldots, x_{s}$ is an $E$-sequence. By Lemma 2.10,

$$
H_{I}(n, E)=H_{I / x_{1} R}\left(n, E / x_{1} E\right)-\left[\left(I^{n+1} E: x_{1}\right) / I^{n} E\right] .
$$

Since $x_{2}, \ldots, x_{s}, x_{1}$ is an $E$-sequence, $\left(I^{n+1} E: x_{1}\right) / I^{n} E=0$. Since $x_{2}, \ldots, x_{s}$ is an $E / x_{1} E$-sequence, the result is now established using the induction hypothesis.

Now we wish to look at the Grothendieck group of a polynomial extension of a ring $R$. Let $R[X]$ be the polynomial extension of $R$ by an indeterminate $X$. Let $\chi$ denote the multiplicity map $\chi_{R[X]}(X)$. Since $R[X] / X R[X]$ is isomorphic to $R$, $\chi: K(R[X]) \rightarrow K(R)$. We have a natural map $\Gamma: K(R) \rightarrow K(R[X])$ induced by the exact functor $\cdot \otimes_{R} R[X]$.

\subsection{Proposition. $\chi \Gamma$ is the identity on $K(R)$.}

With the aid of Proposition 3.2 we give an example of an ideal $I$ and a module $E$ where reduced degree $P_{I}^{*}(n, E) \neq$ degree $P_{I}^{*}(n, E)$. Let $Q$ be the rationals and let $R=Q[y, z]$ subject to the relation $y^{2}+z^{2}=1$. Then $R$ is a Dedekind Domain but is not a Unique Factorization Domain. In fact, $(y, z-1)$ is a prime ideal in $R$ which 
is not principal. For a Dedekind Domain, $K(R)$ is isomorphic to $Z \oplus C(R)$, where $C(R)$ is the ideal class group of $R\left[3, \S 4, \mathrm{n}^{\circ} .8\right]$. This implies that $[R /(y, z-1)]$ is a nonzero element of $K(R)$. However $[R /(y, z-1)]$ belongs to the subgroup $Z(R)$ of $K(R)$, where $Z(R)$ is isomorphic to $C(R)$. Now consider the ring $R[X]$ and let $I=X R[X]$. Let $E=R /(y, z-1) \otimes_{R} R[X]$. Using the notation above, $\chi[E]=$ $[R /(y, z-1)] \neq 0$ in $K(R)$. Since $\chi$ is just the multiplicity map, $\chi[E]=\Delta P_{I}^{*}(n, E)$, which implies the degree of $P_{I}^{*}(n, E)$ is one. But, since $\chi[E]$ is in $Z(R)$, the reduced degree of $P_{I}^{*}(n, E)$ is zero.

\section{REFERENCES}

1. M. Auslander and D. Buchsbaum, Codimension and multiplicity, Ann. of Math. 68 (1958), 625-657.

2. - Corrections to codimension and multiplicity, Ann. of Math. 70 (1959), 395-397.

3. N. Bourbaki, Algèbre commutative, Hermann, Paris, 1961, Chapter 4.

4. J. A. Eagon and M. M. Fraser, A note on the Koszul complex, Proc. Amer. Math. Soc. 19 (1968), 251-252.

5. T. Myata, A remark on M-sequences, Sûgaku 15 (1963-1964), 215-216.

6. D. G. Northcott, Ideal theory, Cambridge Univ. Press, New York, 1953.

7. J. P. Serre, Algèbre locale. Multiplicitiés, Springer-Verlag, New York, 1965.

8. D. Wright, General multiplicity theory, Proc. London Math. Soc. 3 (1965), 269-288:

UNIVERSITY OF ILLINOIS,

URBANA, ILLINOIS 